\title{
Application of Particle Image Velocimetry to a Transonic Centrifugal Compressor
}

\author{
Melanie Voges ${ }^{1}$, Manfred Beversdorff ${ }^{1}$, Chris Willert ${ }^{1}$, Hartmut Krain ${ }^{1}$ \\ 1: German Aerospace Center (DLR), Institute of Propulsion Technology, 51170 Cologne, Germany \\ email: melanie.voges@dlr.de
}

\begin{abstract}
A variety of measurement techniques have been applied to investigate the flow phenomena occurring in advanced turbomachinery components. In an ongoing research project the performance and internal flow field of a high pressure ratio centrifugal compressor is being investigated. Based on the results obtained the compressor was redesigned and resulted in an improved impeller and diffuser. The stage was designed for a total pressure ratio of $6: 1$ and a mass flow rate of $2.55 \mathrm{~kg} / \mathrm{s}$, the corresponding rotor tip speed was $586 \mathrm{~m} / \mathrm{s}$, resulting in transonic flow conditions at the inlet of the diffuser vane passage at a static temperature of $260^{\circ} \mathrm{C}$. The improvement of the impeller flow and the broadening of the stable operating range was predicted by CFD and successfully verified by means of laser-2-focus (L2F) measurements.

Current activities in the research program involve the use of particle image velocimetry (PIV) to analyze and further improve the understanding of the complex flow phenomena inside the vaned diffuser. The study includes phase-resolved measurements of the flow inside a diffuser vane passage with respect to the impeller blade position. Both, instantaneous and phase-averaged velocity fields will be presented.

Optical access is given via a quartz glass window, which was manufactured to match the 3-D contour of the diffuser casing in order to minimize disturbance of the near-wall flow. An adjustable periscope light sheet probe was specifically designed for this application and delivered the laser light sheet (LLS) to the desired vane span positions for the flow field investigation. The periscope probe was purged with compressed air during operation for cooling and to protect the open outlet of the probe from seeding particle deposits.

The flow field results obtained with PIV will be used for validation of the CFD calculations. Based on this approach a better understanding of the complex flow characteristics is expected, which will finally result in a further improvement of the compressor performance.
\end{abstract}

\section{Introduction}

The requirements on modern jet engines' performance, compactness and operating costs continuously increased during the last decades. A prerequisite for a further improvement of today's highly developed engines is a better understanding of the complex internal flow structure of these machines. Reliable information of this type can be obtained by flow visualization provided access to the internal flow field is ensured. A variety of measurement techniques have been applied to investigate the flow phenomena occurring in advanced turbomachinery components. In many cases the planar, laser-based measurement technique particle image velocimetry (PIV) was chosen, as it provides a powerful tool to obtain instantaneous and averaged velocity fields with high spatial resolution in a short time. PIV is also capable of detecting small-scaled turbulent structures and high velocity gradients.

As shown in the past, the application of PIV to centrifugal compressors provides results of good spatial resolution even in transonic flow conditions (Wernet 1999, 2000). More recently, even stereoscopic PIV (SPIV) has been applied to axial compressor flows (Wernet et al. 2005, Liu et al. 2006), but has been focused on large-scale test rigs and low-speed operating conditions. Woisetschläger et al. (2003) showed that in combining PIV with other non-invasive techniques, here laser vibrometry, it is possible to investigate pressure fluctuations in turbine wake flow together with the corresponding phase-resolved velocity fields. Latest developments in PIV 
hardware, such as lasers and cameras, as well as software improvements (e.g. Willert 2004, Scarano et al. 2005, Wernet 2005) support the progress to make this measurement technique available for the increasing demand in high speed applications.

In the framework of an ongoing research program various flow diagnostic and calculation tools have been combined to investigate the performance and the flow field of a high pressure ratio centrifugal compressor. Based on the results obtained the stage was re-designed. An improved impeller as well as an advanced diffuser was applied. The impeller was designed with splitter blades. Its inlet section, the meridional contour and the blade shape were changed such that the impeller exit flow became more homogeneous than before. The diffuser was adjusted to the new impeller exit flow conditions, which implied a conical shape of the diffuser section.

In the following investigations on the test rig the improved stage efficiency and characteristic was experimentally verified, as predicted by CFD calculations. The improvement of the flow field inside the impeller, which was predicted to be more homogeneous, was successfully verified by means of L2F measurements (Förster et al. 2000, Krain et al. 2001 and Krain 2003). For investigation of the flow phenomena in the diffuser region PIV was chosen, as it provides all attributes needed to support and validate the CFD investigations of the compressor flow, which are performed in parallel to the experimental part of the research program.

\section{Experimental Setup}

\subsection{Centrifugal Compressor Stage}

The centrifugal compressor stage was designed for a pressure ratio of 6:1, with a mass flow rate of $2.6 \mathrm{~kg} / \mathrm{s}$ and a rotational speed of 50,000 rpm. The corresponding impeller exit speed is $586 \mathrm{~m} / \mathrm{s}$. This results in transonic flow conditions at the inlet of the diffuser passage, related to static temperatures of $260^{\circ} \mathrm{C}$. Depending on the operating conditions the compressor reaches different temperature levels. In the relevant operating range during PIV investigation the temperatures of casing and flow inside the stage reached $110^{\circ} \mathrm{C}$ up to $255^{\circ} \mathrm{C}$. The impeller consists of 13 main blades and 13 splitter blades in a backsweep design. The impeller exit radius is $112 \mathrm{~mm}$, while the whole stage has a diameter of $600 \mathrm{~mm}$. The diffuser is equipped with 23 profiled guide vanes; the leading edge is positioned at a radius ratio of 1.15 related to the impeller exit. The height of the diffuser passage is $8.1 \mathrm{~mm}$ and remains constant for the whole passage.

\subsection{PIV Setup and Facility Preparation}

The compressor casing can be equipped with a variety of different measurement techniques, such as static pressure and temperature probes, small quartz glass windows for point-based laser diagnostics in the impeller region (L2F) and microphone arrays for acoustic investigations (Fig. 1a). To provide sufficient optical access for planar PIV measurements a large quartz window was needed in the diffuser casing. The prepared access port provided a camera observation area of one complete diffuser vane passage, including the impeller exit region (Fig. 1b). A quartz window and a metal window support were manufactured with considerable effort to precisely fit the inner contour of the diffuser casing, thereby minimizing disturbances of the near-wall flow. The window was milled from a full quartz block and manually surface polished afterwards. The bulky design of the window helps to withstand the high temperatures during compressor operation and to avoid fracture of the glass. Compressive stresses caused by mechanical contact of the glass to the diffuser vanes were considered to be the most likely cause for any damage to the glass. To reduce this strain and to provide a reliable seal between the vane passages a silicone sealing was applied to the contact surface of the vanes in the window area. The window itself was set back in the metal support with a clearance of $0.5 \mathrm{~mm}$, although the resulting edge could interfere with the flow close to the casing. In 
the observed flow area the height of the diffuser passage increased to $8.6 \mathrm{~mm}$ over the entire chord length. Due to the contour of the inner glass surface a certain lens character of the window has to be taken into account. The outer surface of the glass was flat.

a)

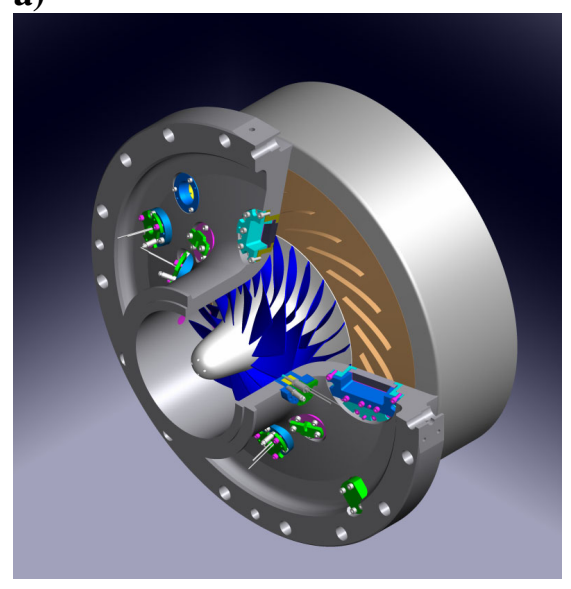

b)

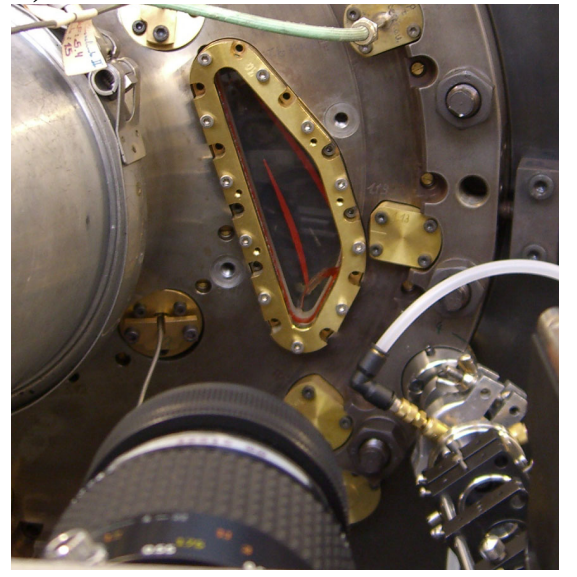

c)

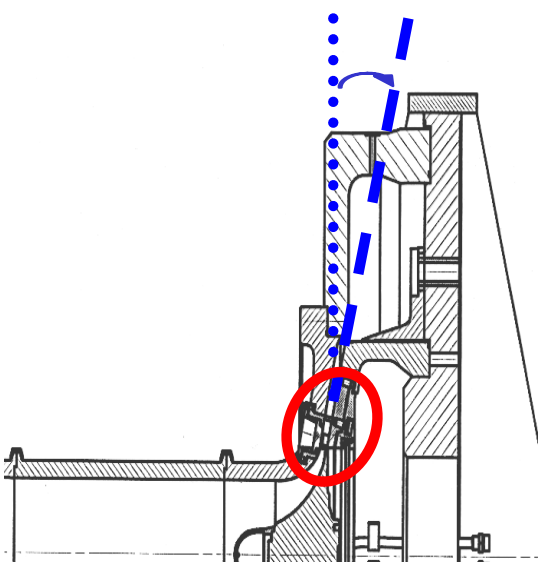

Fig. 1 Compressor facility and PIV set up; a 3D-view of the improved centrifugal compressor stage with casing and instrumentation for flow field and pressure diagnostics; b photograph of camera optical access in diffuser casing and LSP insertion position; c Schematic of the compressor stage giving the new diffuser geometry

For flow observation a thermo-electrically cooled PIV CCD camera with a spatial resolution of $1600 \times 1200 \mathrm{px}$ at a frame rate of $15 \mathrm{~Hz}$ is used. The benefit of the high frame rate is that laser and camera can run at the same frequency which reduces measurement time and therefore saves operating cost of the test facility. Additionally it is possible to run the laser at its design frequency, where the optimum beam profile is achieved. The camera is mounted on a Scheimpflug adapter to optimize alignment of the camera optics with the laser light sheet (LLS). The focal length of the chosen camera lens is $f=105 \mathrm{~mm}$. A precise calibration target is used to align the LLS plane with the PIV camera object plane. The target was made of a $1.9 \mathrm{~mm}$ aluminum plate with a precise $2.5 \mathrm{~mm}$ dot grid applied to one surface. It can be fixed precisely and reproducible in the diffuser vane passage. Adjustment of the grid level in the vane passage is performed with three microscrews.

Due to the conical diffuser geometry (Fig. 1c) it was not possible to use standard $90^{\circ}$ beam deflecting light sheet probes (LSP). Therefore a periscope LSP was specifically designed for this application. The periscope probe allows for adjustment of the LLS in rotational and axial position and angle relative to the chord of the vane profile. Together with the precise probe support in the diffuser casing it is possible to adjust the LLS to the vane span locations chosen for flow investigation. The probe support close to the diffuser outlet is not perpendicular to the outer machine casing, but inclined to adapt to the conical diffuser area. The outer diameter of the periscope probe is $12 \mathrm{~mm}$; the free beam path inside the probe has a diameter of $6 \mathrm{~mm}$. A pair of cylindrical lenses inside the probe form the LLS with a thickness of $1 \mathrm{~mm}$ and a divergence angle of about $6^{\circ}$. At the outlet of the periscope probe a mirror deflects the LLS with an angle of $97^{\circ}$, thus to support the adjustment in the diffuser vane passage. Before entering the LSP the beam diameter of the frequency-doubled, double-cavity $120 \mathrm{~mJ}$ Nd:YAG PIV laser has to be reduced to pass through the periscope probe without streaking the metal surface of the inner tube. Here a pair of spherical lenses are used in a telescopic set up. The optical path of the laser beam through the periscope and the related lens set up is illustrated in Fig. 2. The laser beam is delivered via an articulated guide arm to the interface of the LSP. The periscope is purged with compressed, dry air to avoid deposition of seeding particles and for cooling purposes, as the beam path at the outlet of 
the periscope probe has not been sealed with a glass window. For synchronization of the laser pulses and camera exposure a programmable sequencer unit is used. In combination with a phase shifter the 1/rev-trigger of the compressor can be used to perform phase-resolved PIV measurements with different phase angle relations between impeller and diffuser vanes.

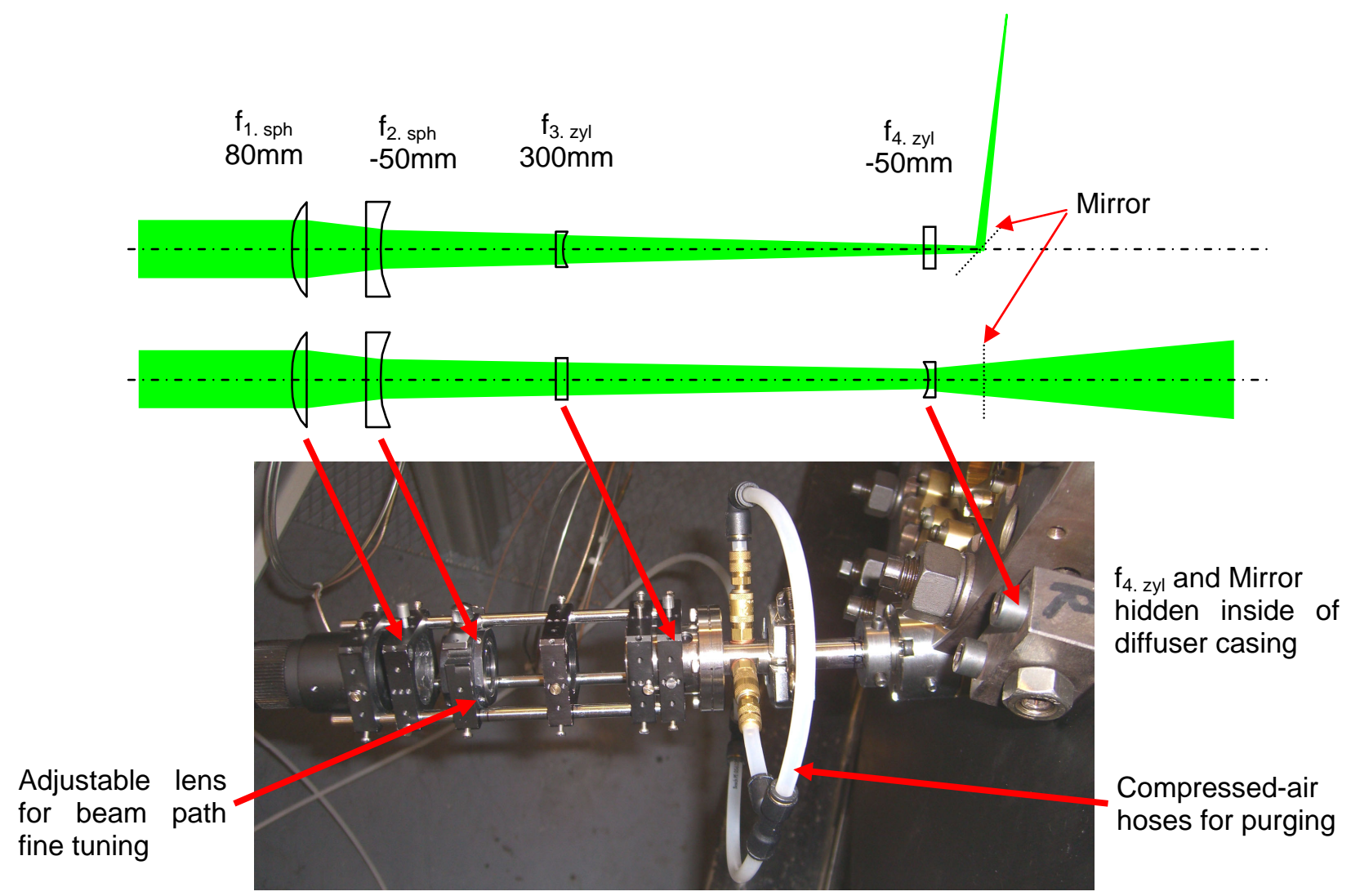

Fig. 2 Setup of periscope LSP with beam path schematic

To add seeding particles to the flow a manifold containing four seeding probes was inserted upstream of the impeller to the inlet tube. The probes are fixed at four radial positions with a small axial displacement to ensure an almost uniform seeding distribution over the intake radius. A circumferential traverse allows for the adjustment of the seeding location in dependence of the compressor operating conditions, in order to optimize the particle concentration at the measurement position. The seeding particles are produced in Laskin-nozzle generators installed in a battery of three in parallel using paraffin oil.

The PIV equipment, including laser head, camera and Scheimpflug adapter as well as a light source for camera calibration, was mounted on a massive support decoupled from the test rig. This was necessary to minimize the transmission of machine vibrations to the PIV hardware during operation. The only hardware interface between test rig and PIV set up was the articulated laser guide arm, which was connected to the beam exit of the laser head and to the LSP.

\subsection{Parameter Studies at Low Flow Conditions}

In advance to the PIV measurements on the chosen operating points of the centrifugal compressor parameter studies were carried out for optimization of the PIV set up. Therefore the camera was equipped with an objective of $55 \mathrm{~mm}$ focal length, allowing for flow observation over the entire chord length of the upper vane (Fig. 3a, left) but at a low image resolution. The compressor was operated under low speed conditions at 35,000 rpm. At this operating point pre-conditioning of the seeding probes was performed, using only one of the four probes. This resulted in a stream tube 
seeding of the flow. As shown in the middle of Fig. 3a, the seeding was not very homogeneous in the light sheet section. This was caused by an improper circumferential alignment of the probe to the flow conditions during the feasibility studies. The visible structures may possibly be wake of the impeller blades or mixing phenomena, such as tip clearance flow in the impeller section, where unseeded flow can mix with the seeded stream lines. Despite of the poor seeding quality the trigger chain of laser and camera was checked concerning a correct phase relation between impeller blades and diffuser vanes. The desired phase angles were verified with the help of an oscilloscope. Processing of the raw data at this early stage during feasibility studies showed surprisingly good results. In the right part of Fig. 3a, an averaged, phase-resolved velocity field is shown, processed with an interrogation window of $48 \times 48 \mathrm{px}$ at $50 \%$ sample overlap. The corresponding grid size was $1.5 \times 1.5 \mathrm{~mm}^{2}$. Due to the low spatial resolution the expected small-scaled turbulent structures could not be detected, but the mean velocity in the light sheet section matches the predicted values of the CFD calculations.
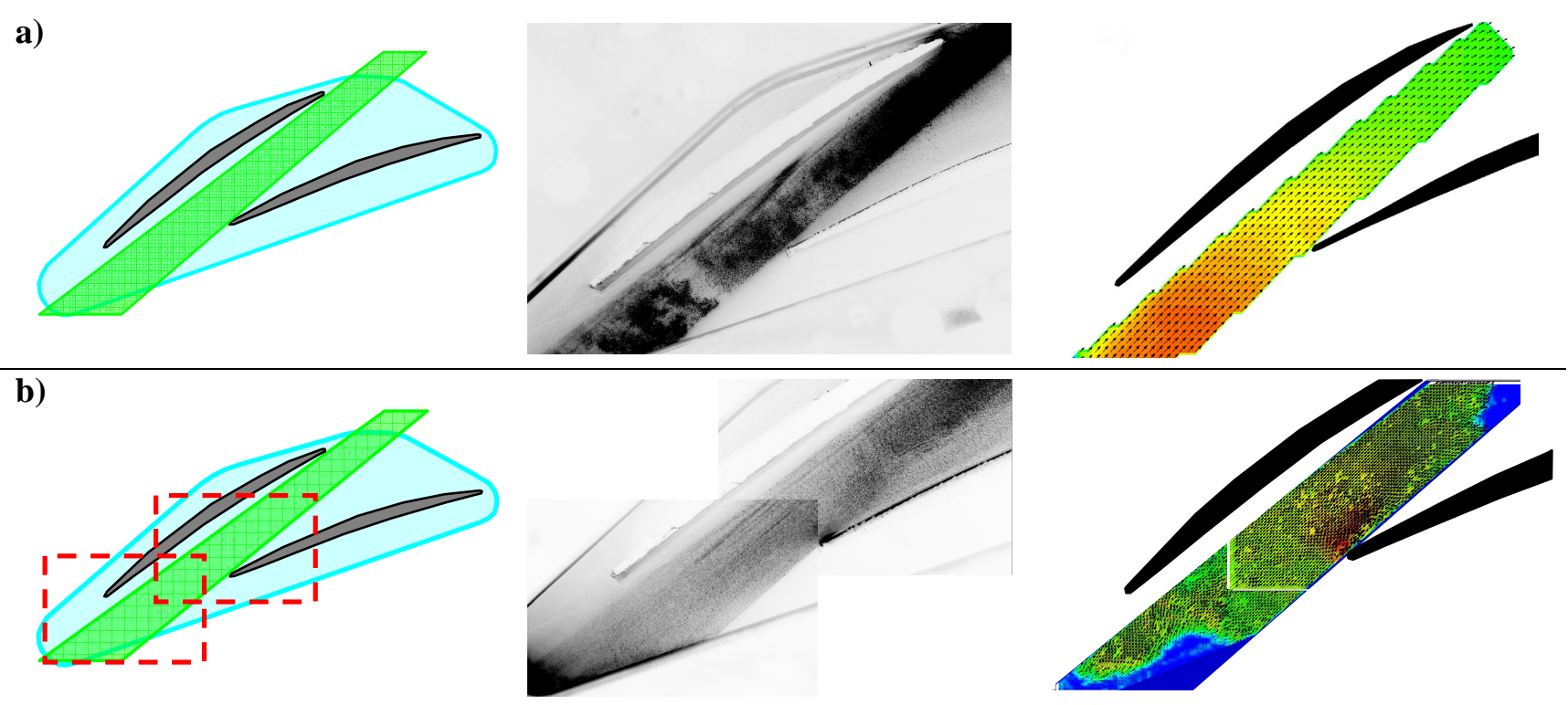

Fig. 3 Optimization of seeding quality and camera and LLS position during parameter studies: a first general camera view with low image resolution; $\mathbf{b}$ final PIV set up with two camera viewing positions and optimized seeding quality

In a next step the camera's spatial resolution as well as the seeding quality was improved using a $f=105 \mathrm{~mm}$ camera lens operating at $f / 4.0$ along with all four seeding probes during parameter studies. The first camera position was chosen to investigate the flow close to the impeller exit; the second camera position followed the flow into the diffuser vane passage. The resulting combined observation areas of the two camera positions with improved seeding quality are shown in Fig. 3b. With the higher resolution optics the flow could be observed over three quarters of the upper vane's chord length in two measurement steps allowing for detailed investigation of small-scaled structures. The use of all four seeding probes resulted in a more homogeneous particle distribution in the illuminated light sheet area. Additionally, the size of the particles could be optimized by switching off the impactor of the Laskin-nozzle-generators. The impactor is used as a kind of obstacle in the particle flow inside of the generator. Particles of a larger size, that are not able to follow the flow precisely, will be collected by the impactor and removed from the seeding so that only particles of a size less than $1 \mu \mathrm{m}$ will leave the particle generator. In switching off the impactor the particle size distribution in the seeded flow can be increased to a certain extend. Taking into account the high temperatures during compressor operation the particles will start to evaporate in the flow field. While the small particles might evaporate, larger particles survive longer at higher 
temperatures although they have reduced their size when reaching the investigated flow area. This had a significant positive effect on the results. Evaluation of one instantaneous PIV image pair applying an interrogation window of $32 \times 32 \mathrm{px}\left(1.2 \times 1.2 \mathrm{~mm}^{2}\right)$ with $50 \%$ overlap and a corresponding grid size of $0.6 \times 0.6 \mathrm{~mm}^{2}$ showed results of very good quality (Fig. 3b, right). This final PIV setup was chosen for the applied test sequence.

\section{PIV Measurement Sequence}

\subsection{Compressor Operation}

For flow investigation with PIV the same four operating points as for the L2F measurements in the impeller region were chosen in order to obtain comparable results with the different measurement techniques. The operating conditions, such as standard day-corrected rotational speed, corrected mass flow as well as the temperature levels reached in the flow field and at the diffuser casing, are summarized in Table 1. The mass flow is determined with the help of a venturi nozzle installed in the inlet pipe considering the daily environmental conditions of ambient temperature and barometric pressure.

Table 1 Operating points chosen for PIV investigation of the compressor flow

\begin{tabular}{l|cccc}
\hline Rotational speed $n_{\text {red }}$ & $35,000 \mathrm{rpm}$ & $44,000 \mathrm{rpm}$ & $50,000 \mathrm{rpm}$ & $50,000 \mathrm{rpm}$ \\
\hline \hline Mass Flow $\dot{m}$ & $1.4 \mathrm{~kg} / \mathrm{s}$ & $2.15 \mathrm{~kg} / \mathrm{s}$ & $2.6 \mathrm{~kg} / \mathrm{s}$ & $2.83 \mathrm{~kg} / \mathrm{s}$ \\
Pressure ratio & $2.5: 1$ & $4.0: 1$ & $5.6: 1$ & $5.3: 1$ \\
Casing Temperature T & $110^{\circ} \mathrm{C}$ & $175^{\circ} \mathrm{C}$ & $245-255^{\circ} \mathrm{C}$ & $230-235^{\circ} \mathrm{C}$ \\
PIV pulse separation & $2.5 \mu \mathrm{s}$ & $2.0 \mu \mathrm{s}$ & $1.5 \mu \mathrm{s}$ & $1.5 \mu \mathrm{s}$ \\
\hline
\end{tabular}

\subsection{Data Acquisition and Post-processing}

In the PIV test sequence phase-resolved measurements were carried out using eight phase angle relations per main-splitter passage. As the impeller exit flow was expected not to be symmetric between main-splitter and splitter-main blade passages, the number of phase angles was doubled keeping the $45^{\circ}$ interval. The resulting 16 phase angle relations $\left(0^{\circ}-720^{\circ}\right)$ allow for detailed flow investigation related to one complete main-splitter-main passage. Per phase angle a total of 188 images was recorded in a PIV sequence. In addition one PIV sequence without seeding was recorded to be able to correct for scattered laser light during image post processing. Three light sheet planes were selected for each operating point of the centrifugal compressor: one close to the hub at $19 \%$, one at mid span level (50\%) and one close to the casing at $74 \%$ passage height. The upper and lower limitation to the LLS position was given due to the amount of stray light being reflected from the hub or the casing surface. The PIV laser was operated at $15 \mathrm{~Hz}$ repetition rate with a pulse separation depending on the rotational speed of the impeller (see Table 1). Flow analysis in the diffuser vane passage could be performed in an illuminated area of $60 \times 16 \mathrm{~mm}^{2}$ per camera view.

Evaluation of the PIV image data was performed after pre-processing with high pass filter, subtraction of background image and masking image areas without velocity information (e.g. diffuser casing or window support). An example for the image pre-processing is illustrated in Fig. 4. Fortunately distortion of the particle images due to the lens character of the quartz window was insignificant. Therefore a dewarping procedure of the images was not necessary. A correlation algorithm with multi-grid option resulting in a final $32 \times 32 \mathrm{px}$ interrogation window with $50 \%$ overlap was used. For the calculation of averaged velocity fields all 188 images of a PIV sequence 
were considered. The re-combination of the obtained velocity fields for both camera views could be easily performed during post-processing of the PIV data with the help of the common calibration grid, as both camera views overlap in one area.

a)

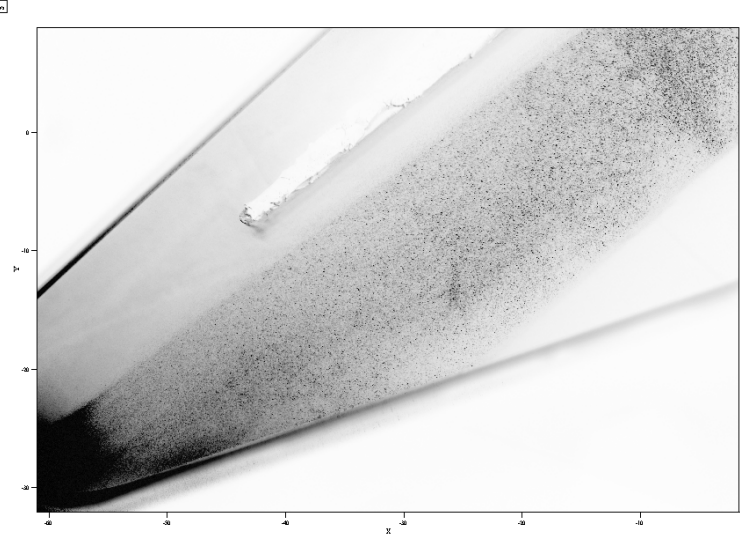

b)

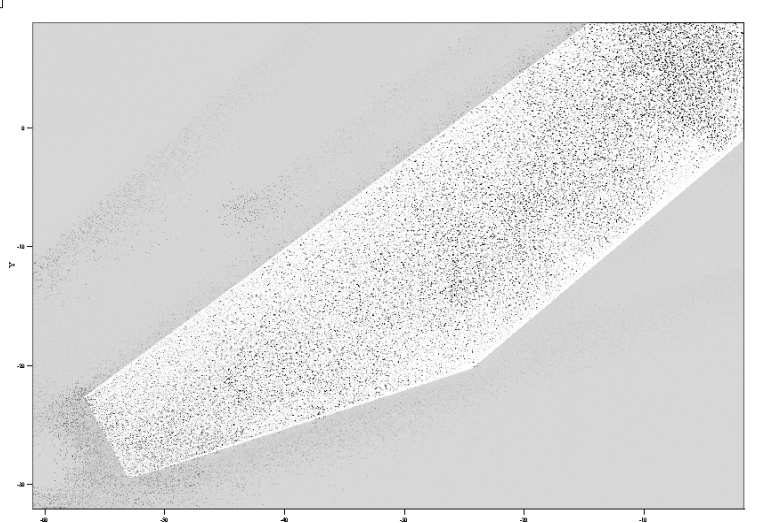

Fig. 4 The effect of image pre-processing from a raw data image with scattered light from impeller blades in the lower left corner to $\mathbf{b}$ pre-processed image with reduced background light, high pass filter and mask, shown for the first camera position (both images are shown with inverted scale)

\subsection{Error Analysis}

To quantify the order of magnitude of possible errors made during PIV data recording and processing, different error sources have to be taken into account (Raffel et al. 1998). According to the approach made by Westerweel (2000) a measurement error of $0.1 \mathrm{px}$ can be assumed. With a mean pixel shift varying from $13 \mathrm{px}$ at $35,000 \mathrm{rpm}$ up to $20 \mathrm{px}$ at 50,000 rpm, this led to a relative measurement error of 0.5 to $0.8 \%$ ( 2.7 to $3.5 \mathrm{~m} / \mathrm{s}$ in the absolute domain). In this case the size of single interrogation areas achieved during processing was $0.6 \times 0.6 \mathrm{~mm}^{2}$. This means that structures were passing at frequencies between $500 \mathrm{kHz}$ and $120 \mathrm{MHz}$ in the measured velocity range of 300 to $700 \mathrm{~m} / \mathrm{s}$. Here the size of the particles had an important influence on the obtained velocity data. As the response time of particles about $1 \mu \mathrm{m}$ in size can be calculated to $10 \mu$ s (Raffel et al. 1998), the particles behave like a low pass filter with a cut off frequency of $100 \mathrm{kHz}$ applied to the flow. Given a blade passing frequency around $20 \mathrm{kHz}$ implies that only large scale structures are faithfully captured, while smaller scales are damped out. Here the use of sub-micron or nano-sphere particles could be considered, but this would have the effect of a significant decrease in light reflection of the particles (Rayleigh scattering).

Another experimental error source for this specific PIV measurement was introduced with the precision of the mechanical set up. A small uncertainty in the adjustment of the two camera positions could not be eliminated. The error made during traversing of camera was $0.5 \mathrm{~mm}$. This is visible in the re-combined velocity maps of both camera views, but has no relevance for each single camera view.

\section{Results and Discussion}

In the following a selection of the results obtained in the PIV measurement campaign is presented. To give an overview of the different operating conditions, the absolute Mach number distribution at $50 \%$ passage height is shown in Fig. 5 . The Mach number calculation was based on the related timeaveraged velocity data sets and the temperatures given in Table 1 . It is obvious that the mean passage velocity is increasing with the impeller rotational speed. An additional velocity variation is visible comparing the three light sheet positions, where the highest velocity can be found at the $50 \%$ 
level in the vane passage (Fig. 6b). The velocity close to the hub at 19\% span level, as shown in Fig. $6 \mathrm{a}$, is reduced by about $50 \mathrm{~m} / \mathrm{s}$, which can be identified as the beginning influence of the hub surface. A significant reduction of the velocity can be observed in the upper LLS plane at $74 \%$ passage height (Fig. 6c). This phenomenon near the casing wall was expected and can be explained with the presence of a wake flow close to the shroud at the impeller exit, which was found by 3Dcalculations and additionally during investigation of the impeller exit flow with the L2F method (Krain et al. 2001).

a)

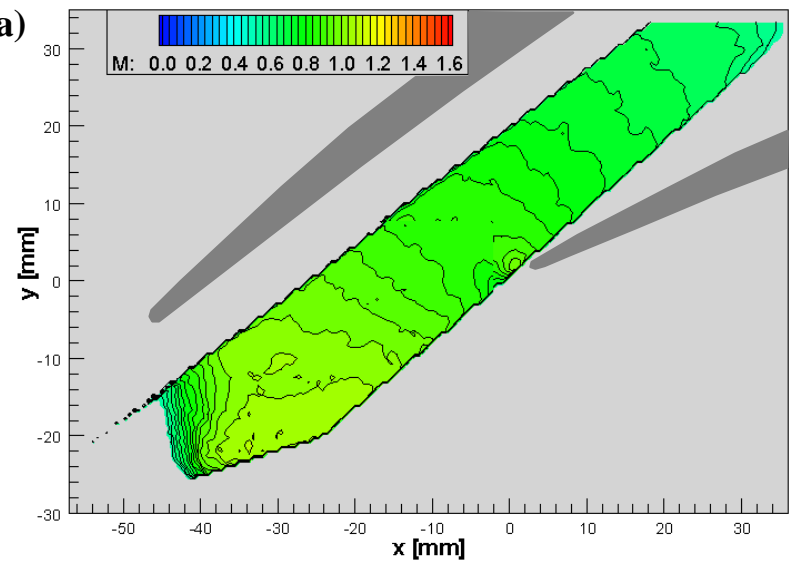

b)

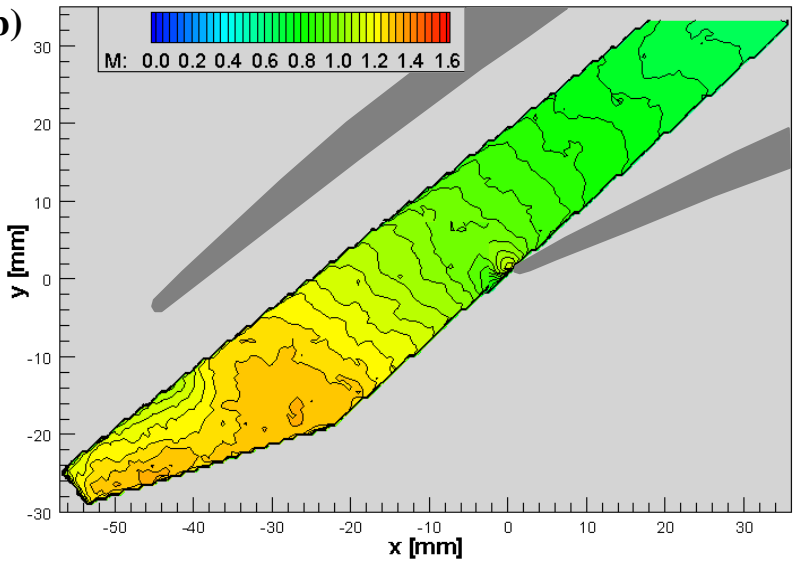

c)

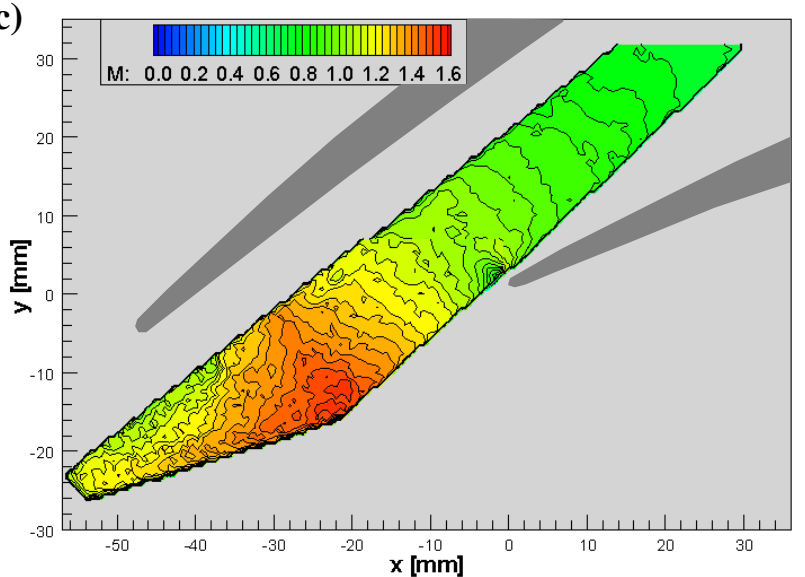

Fig. 5 Mach number distribution at 50\% diffuser passage height for different operating conditions of the compressor: a 35,000 rpm; b 44,000 rpm; c 50,000 rpm (mass flow $=2.6 \mathrm{~kg} / \mathrm{s}$ )

Following the development of the transient diffuser passage flow obtained with the different phase angle relations, the flow structures of the passing impeller blades become visible. At a rotational speed of 50,000 rpm the impeller blades were passing at a frequency of $21.74 \mathrm{KHz}$. Based on the exit speed of $580 \mathrm{~m} / \mathrm{s}$ the flow patterns, such as wake structures or blade passage flow packages, followed each other at a time interval of $46 \mu \mathrm{s}$, resulting in a spatial distance of $26.7 \mathrm{~mm}$. Such structures can be identified in the phase-resolved, unsteady velocity fields, as illustrated in Fig. 7. Only one example is shown herein, as the evaluation of the PIV data was still in progress while this paper was being prepared. The markers noted with a point out wake structures behind the blades. Due to the proximity to the impeller exit the wake appears much stronger in the lower left corner of the image than downstream in the diffuser inlet area. Here the mixing of blade passage flow structures, marked with $\mathbf{c}$ in Fig. 7, and the wake is already smoothening the flow patterns. 
a)

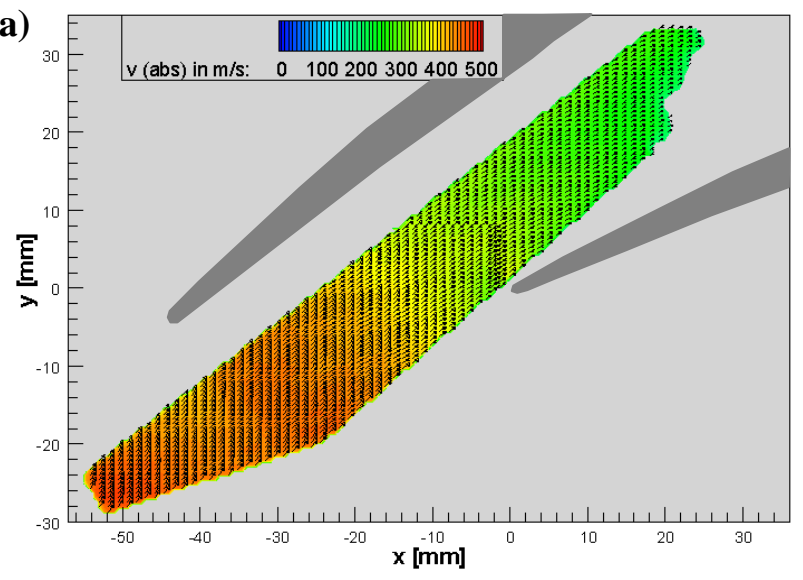

b)

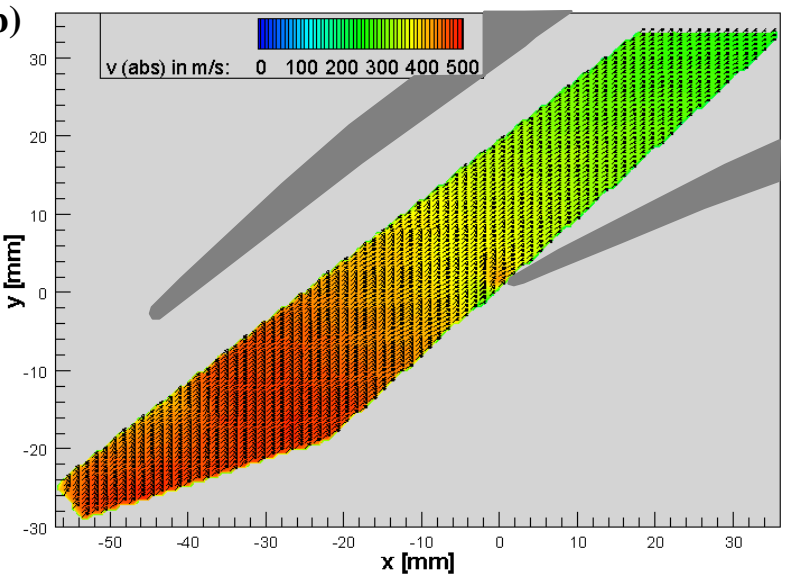

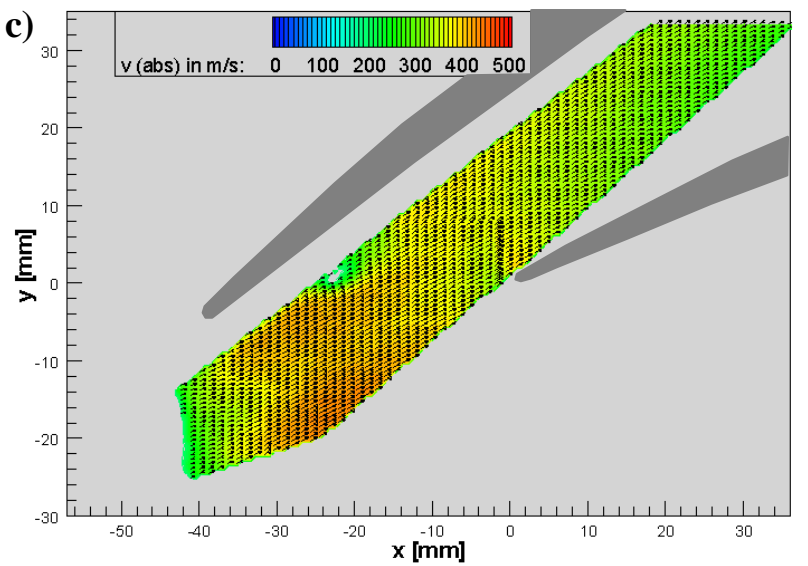

Fig. 6 Time-averaged velocity maps at a rotational speed of 44,000 rpm for different positions of the Light sheet plane: a 19\% passage height (close to the hub); b 50\% passage height; c 74\% passage height (close to the casing)

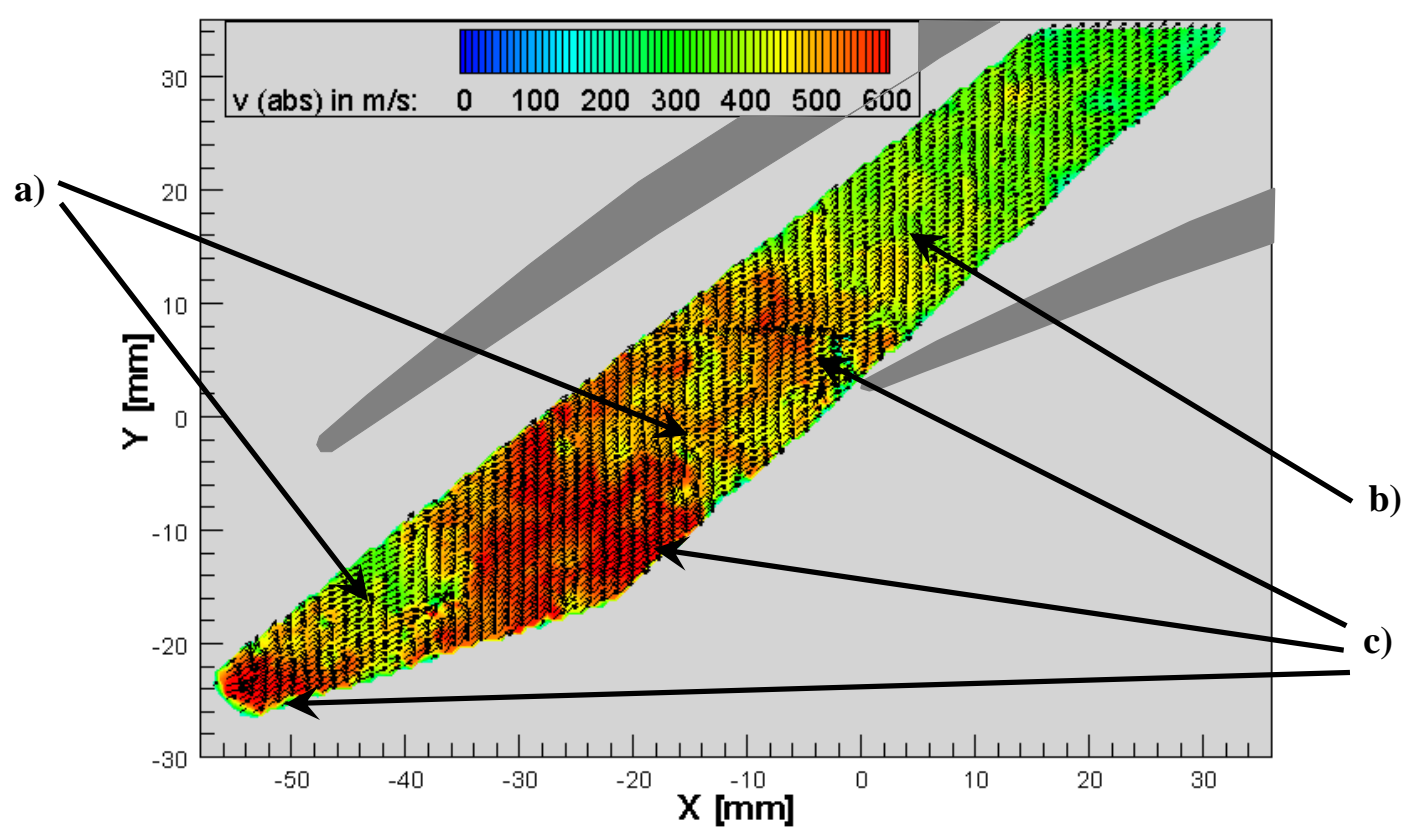

Fig. 7 Unsteady phase-resolved velocity map at 50\% span level and 50,000 rpm for one phase relation $\left(0^{\circ}\right)$ within a main-splitter-main cycle; a wake structures of main and splitter blade, $\mathbf{b}$ mixing flow in the diffuser vane passage, $\mathbf{c}$ blade passage flow 


\section{Outlook on Stereo-PIV}

Subsequent to the PIV test program the setup was extended with an additional camera and Scheimpflug adapter to arrive at a stereoscopic setup in an effort to recover the full threecomponent velocity field. The cameras were arranged symmetrically with one camera observing the light sheet in forward scatter while the other operated in back-scatter mode. Calibration of the stereoscopic setup was performed on single images of the planar calibration target as it was not possible to calibrate the field of view within the vane passage using a translated or multilevel target. This camera model based calibration, described in detail in Willert (2006), recovered the camera positions and hence respective viewing angles of $\pm 23^{\circ}$ with respect to the light sheet. A disparity correction based on the actual particle image data accounted for a remaining misalignment of 10$20 \mathrm{px}(0.4-0.8 \mathrm{~mm})$ of the camera views with respect to each other. Following the calibration an additional test run with SPIV was carried out by acquiring phase-resolved measurements at a rotational speed of $35,000 \mathrm{rpm}$. The convincing results encouraged to further extend the measurement program by running a complete phase-resolved SPIV sequence at 50,000 rpm.

A detail from the flow field showing an accelerated blade passage structure at the diffuser inlet is given in Fig. 8. To emphasize the turbulent behavior of the flow a mean value of $|U|=470 \mathrm{~m} / \mathrm{s}$ has been removed from the images and from the velocity data. For both individual camera viewing directions the same flow structures can be identified for the in-plane velocity components. In the particle images shown in the lower part of Fig. 8 both camera frames are overlaid to visualize that the dominating flow phenomena are already visible and can be recovered in both views.
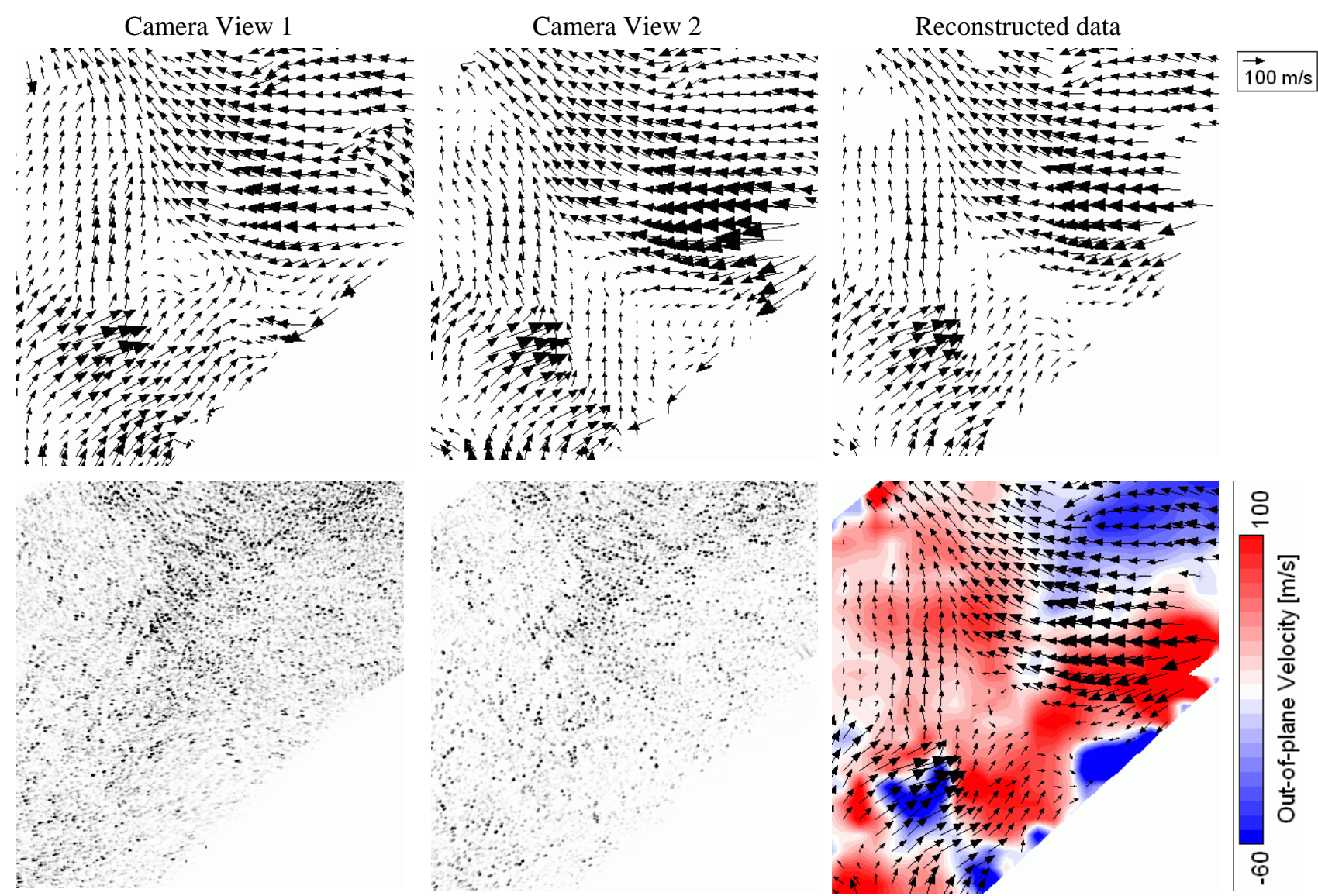

Fig. 8 Flow details of the SPIV measurement at 50\% span level and 50,000 rpm for both camera views, showing vector plots and particle images and the resulting out-of-plane velocity. Camera View 1 is arranged in forward scatter with respect to the light sheet. 
Assuming a displacement measurement error of $0.1 \mathrm{px}$ on the PIV data for each camera, the measurement uncertainty of the recovered 3-C velocity data can be estimated to be 2 and $1.5 \mathrm{~m} / \mathrm{s}$ for the in-plane velocity components and about $3 \mathrm{~m} / \mathrm{s}$ for the out-of-plane velocity. The 3-C velocity data presented in Fig. 8 contains only those velocity vectors for which the residuals in the 3-C reconstruction from the four measured displacements are less than $1 \mathrm{px}(20 \mathrm{~m} / \mathrm{s})$. This shows the necessity of proper reconstruction prior to interpreting any of the acquired results.

In general the reconstruction of the recorded SPIV data showed very good results, provided that the seeding quality was homogeneous and background stray light could be reduced to a minimum.

\section{Summary}

In this study the planar PIV technique was successfully implemented to the vaned diffuser passage of a new high pressure ratio transonic centrifugal compressor. Prior to the experimental part of the research program specific test equipment was designed, such as a periscope LSP and a quartz window for optical access. Measurements were performed at different operating conditions of the centrifugal compressor. For flow investigation in the diffuser vane passage three light sheet positions at different span levels were chosen. To achieve a high spatial resolution the camera was traversed, allowing for a detailed observation of the impeller exit flow and the development of the flow into the diffuser passage. To support and validate the CFD calculations in a parallel part of the research program, phase-resolved measurements were performed. The results obtained are of high quality, as first evaluations have confirmed. An overview on the velocity distribution in the diffuser vane passage was given, as well as a brief discussion of the phase-resolved results. Both, averaged and instantaneous velocity fields were presented. The PIV data base established in this program can be taken as basis for further detailed evaluations of the flow phenomena occurring in the centrifugal compressor stage. Based on the combined results of PIV measurements and CFD calculations a better understanding of the complex flow characteristics is expected for the future.

In extension to the main part of the measurement program a SPIV setup was realized at the test rig with the successful demonstration of this advanced measurement technique in the enclosed environment of the diffuser passage. This additional data set may give an important contribution to the understanding of turbulent flow development in the impeller-diffuser-interaction.

\section{Acknowledgments}

The project was sponsored by the German Ministry of Economy via AIF and FVV (BMWi/AIF-No. 13228/N1, FVV-No. 067980). The authors would like to thank these organizations as well as the industrial partners for the permission to publish the results presented in this paper. We also like to thank the technical personnel of the compressor facility for their support during the test campaign.

\section{References}

Förster W, Karpinski G, Krain H, Röhle I., Schodl R (2000): 3 Component, Doppler Laser-2-Focus velocimetry applied to a transonic centrifugal compressor. 10th International Symposium on Applications of Laser Techniques to Fluid Mechanics, Lisbon, Portugal, July 10-13

Krain H, Karpinski G, Beversdorff M (2001): Flow analysis in a transonic centrifugal compressor rotor using 3-component laser velocimetry. 2001-GT-0315. Proceedings of ASME Turbo Expo 2001. New Orleans, Louisiana, USA, June 4-8

Krain H (2003): Review of centrifugal compressor's application and development. GT2003-38971. Proceedings of ASME Turbo Expo 2003. Atlanta, Georgia, USA, June 16-19

Liu B, Yu X, Liu H, Jiang H, Yuan H, Xu Y (2006): Application of SPIV in turbomachinery. Exp Fluids 40: 621-642

Raffel M, Willert C, Kompenhans J (1998): Particle image velocimetry, a practical guide. Springer, Berlin 
Scarano F, David L, Bsibsi M, Calluaud D (2005): S-PIV comparative assessment: image dewarping and misalignment correction and pinhole and geometric back projection. Exp Fluids 39: 257-266

Wernet MP (1999): Application of digital particle image Velocimetry to turbomachinery. Lecture Series on Planar optical measurement methods for gas turbine components. RTO-EN-6 AC/323(AVT)TP/20. Cranfield, UK, 16-17 September and Cleveland, USA, 21-22 September

Wernet MP (2000): Application of DPIV to study both steady state and transient turbomachinery flows. Optics \& Laser Technology 32: 497-525

Wernet MP (2005): Application of planar Velocimetry in high speed flows: state-of-the-art and perspectives. PIVnet II International workshop on the application of PIV to compressible flows. Delft, The Netherlands, June 6-8

Wernet MP, van Zante D, Strazisar TJ, John WT, Prahst PS (2005): Characterization of tip clearance flow in an axial compressor using 3-D digital PIV. Exp Fluids 39: 743-754

Westerweel J (2000): Theoretical analysis of the measurement precision in particle image velocimetry. Exp Fluids 29: Suppl. S3-S12

Willert C (2004): Application potential of advanced PIV algorithms for industrial applications. PIVnet/ ERCOFTAC workshop on particle image Velocimetry. Lisbon, Portugal, July 9-10

Willert C (2006): Assessment of camera models for use in planar velocity calibration. Exp Fluids (submitted and accepted, to appear in 2006)

Woisetschläger J, Mayrhofer B, Hampel B, Lang H, Sanz W (2003): Laser-optical investigation of turbine wake flow. Exp Fluids 34: 371-378 